\title{
Smell and Taste Disorders in COVID-19 Patients: Objective Testing and Magnetic Resonance Imaging in Five Cases
}

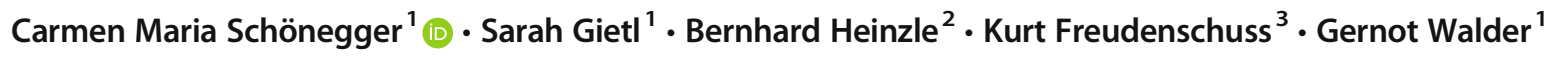

Accepted: 19 October 2020 / Published online: 24 October 2020

(C) Springer Nature Switzerland AG 2020

\begin{abstract}
Smell and taste disorders are acknowledged as characteristic symptoms for SARS-CoV-2 infection by now. These symptoms have been linked to a neuroinvasive course of disease. In this study, we investigated five consecutive COVID-19 patients with a prolonged course of dysosmia and dysgeusia. Those with objectifiable alteration in taste or smell were subjected to MRI with contrast agent to investigate possible involvement of the central nervous system. We found dysosmia and dysgeusia to be mostly objectifiable, but no evidence for neuroinvasiveness could be detected by MRI in the late stage of the disease. Alterations in taste and smell could be objectified in most patients. Nevertheless, no evidence for a neuroinvasive potential could be identified by MRI, at least in the late stage of disease. We encourage medical professionals to conduct specialized examinations and MRIs in the acute stage of disease, which guarantees an optimum patient care.
\end{abstract}

Keywords Smell and taste disorders $\cdot$ Objective olfactory and gustatory testing $\cdot$ MRI $\cdot$ Neuoinvasive potential

\section{Introduction}

Although a lot of effort has been made in order to investigate this novel coronavirus, our knowledge about many pathogenic aspects of COVID-19 is still limited. Clinical and experimental studies proved that several coronaviruses have neuroinvasive capacities, since they show the ability to spread from the respiratory tract to the central nervous system [1]. Hypothesis of neurotropism of SARS-CoV-2 is based on COVID-19 patients with neurological manifestations. Furthermore, neurological complications up to actual damage hint neurovirulence [2]. Actual case reports substantiate this assumption. COVID-19 can cause meningitis with a fatal

Carmen Maria Schönegger and Sarah Gietl contributed equally to this work.

This article is part of the Topical Collection on COVID-19

Carmen Maria Schönegger

c.schoenegger@campus.lmu.de

1 Department of Virology, Dr. Gernot Walder GmbH, 9931 Außervillgraten 30, Austria

2 Radiology Private Clinic Kursana, Wörgl, Austria

3 HNO-CHIRURGIE-TIROL.com, Hochrum, Lienz, Austria outcome, which was evidenced by the case of a 24-year-old man in Japan, who succumbed to the disease [3]. Brain MRI demonstrated hemorrhagic lesions that were consistent with acute necrotizing hemorrhagic encephalopathy [4].

Another manifestation of nervous system involvement is the appearance of alterations of taste and smell [5]. Anosmia and dysgeusia are by now acknowledged as significant symptoms in association with COVID-19 by the American Academy of Otolaryngology-Head and Neck Surgery (AAO-HNS) and other researches [6, 7]. Case series show a high frequency of chemosensitive disorders in patients, ranging between 19.4 and $88 \%[8,9]$. According to Vaira et al., the presence of olfactory and gustatory dysfunction may predict a milder course of disease. On the other hand, the neglect of such symptoms suggests a more severe course of disease [10]. Some patients reported isolated olfactory or taste disorders, but more complained about a combined dysfunction. Even though a complete recovery could be noted in regard to most patients, evidence has been found about persisting alterations in chemosensory function for a prolonged period [11].

At the Shahid Beheshti University of Medical Sciences in Tehran/Iran, possible olfactory bulb alterations were investigated by olfactory bulb magnetic resonance imaging. In spite of isolated sudden onset anosmia and positive SARS-CoV2 polymerase chain reaction during the acute phase of the 
disease, the MRI demonstrated normal volume and signal intensity of olfactory bulb with no sign of nasal congestion [12].

\section{Methods}

\section{Enrolment and Data Collection}

The study was conducted on five patients reporting impairment of taste and smell with confirmed SARS-CoV-2 infection in East Tyrol. Written informed consent was obtained from all participants. Age ranged between 41 and 57 years, and all the participants were female. Clinical data and history of symptoms were assessed anamnestically.

Olfactory and gustatory testing was conducted between 11 and 30 days after disease onset, which was defined as the commencement of symptoms. In case objective testing revealed alterations in taste or smell, patients were subjected to MRI in a timely manner. A follow-up examination was conducted in three of the patients whose MRI raised suspicion of alterations in the first run.

\section{Olfactory and Gustatory Function Assessment}

Olfactory and gustatory function assessment was carried out by means of Burghart Screening 12 Test with taste strips in accordance with the manufacturer's instructions $[13,14]$.

In order to evaluate olfactory function, an identification test, called Sniffin' Sticks, was performed [13]. Twelve common odorants were consecutively presented to the patient close to both nostrils. Out of four options the participant was requested to identify the presented smell. Results were interpreted according to a scoring system considering patients age. A score above ten was determined as normal, between six and ten a hyposmia was diagnosed and a score below six was reported as anosmia.

Gustatory testing was performed by taste strips covering the four basic gustatory qualities sweet, salty, sour, and bitter [14]. Taste strips were applied on patient's tongue. For each stripe, the test person had to choose the corresponding odor from four answers. According to the count of correct answers, four categories of classification were established: normal (score 4), mild hypogeusia (score 3), moderate hypogeusia (score 2), severe hypogeusia (score 1), and ageusia (score 0).

\section{Magnetic Resonance Imaging}

A standard brain MRI protocol was used involving axial T2 TSE, axial FLAIR, axial DWI (b0 and b1000), dADC, axial T2w FFE, and isotropic T1w 3D IR TFE with a 1-mm slice thickness without contrast media and ceT1w 2D MPRAGE, on a 1.5T Prodiva Philips MRI machine. The 3D T1 sequences with and without contrast were reconstructed in coronal, axial, and sagittal projection for the radiologic evaluation. A short-term follow-up examination 10 days later was performed with the same axial FLAIR and DWI/dADC. Sequences were used as well as isotropic 3D T2 and 3D FLAIR sequences (Appendix).

\section{Results}

Olfactory and gustatory testing was conducted between 11 and 30 days after onset of disease, which was defined as the commencement of symptoms. Out of the five patients three showed a manifest anosmia, one result was rated as a hyposmia and one as normosmia. Gustatory testing revealed two normal scores (4/4), one moderate hypogeusia (2/4), one severe hypogeusia (1/4), and one ageusia (0/4). Detailed results are summarized in Table 1 .

Three weeks after the first olfactory and gustatory examination, a follow-up testing was conducted with the three patients who also had a second MRI. This investigation revealed one persistent anosmia (5/12), one persistent hyposmia (7/12), and one patient's condition improved from anosmia to hyposmia (10/12). Gustatory testing showed an enhancement in two patients $(3 / 4 ; 4 / 4)$; one patient had the same score $(4 / 4)$. Detailed results are summarized in Table 2.

None of the patients showed any changes in the paranasal sinuses, not even small fluid collections or mucosal swelling. The cribriform plate was normal in all cases, and the olfactory nerve was normal in size and signal, without any side differences and there was no neuronal or perineural enhancement. The meninges showed no enhancement or thickening. The gyrus rectus was normal in size and signal intensity in all patients and showed no swelling.

No signal changes in the medial temporal lobes or the thalami could be found and no hemorrhagic microbleeding in T2* sequences were observed. Even though ischemic events and leptomeningeal enhancement have already been reported, there were no signs for an acute or chronic ischemic event [15].

Table 1 Results of the first gustatory and olfactory testing

\begin{tabular}{lll}
\hline & Olfactory testing & Gustatory testing \\
\hline Patient 1 & $4 / 12$ & $4 / 4$ \\
Patient 2 & $5 / 12$ & $2 / 4$ \\
Patient 3 & $6 / 12$ & $0 / 4$ \\
Patient 4 & $8 / 12$ & $1 / 4$ \\
Patient 5 & $12 / 12$ & $4 / 4$ \\
\hline
\end{tabular}


Table 2 Results of the second gustatory and olfactory testing

\begin{tabular}{lll}
\hline & Olfactory testing & Gustatory testing \\
\hline Patient 1 & $5 / 12$ & $4 / 4$ \\
Patient 2 & $10 / 12$ & $4 / 4$ \\
Patient 4 & $7 / 12$ & $3 / 4$ \\
\hline
\end{tabular}

In the first examination, one patient showed a symmetric, slightly hyperintense signal in the head of the caudate nucleus, parahippocampal gyrus, and the uncus, predominantly on the left side. In T2, the area appeared thicker and swollen with a higher SI, but all the other sequences including DWI were normal. Also, SI measurements were slightly elevated with values of 1021 compared to 865 on average in primarily healthy individuals. In the follow-up examination, there was a slight drop to 970 , but the additional high-resolution 3D FLAIR and T2 images showed no anomalies, so probably this finding lies within the normal range. All the other patients had a normal brain MRI (Images 1, 2, and 3).

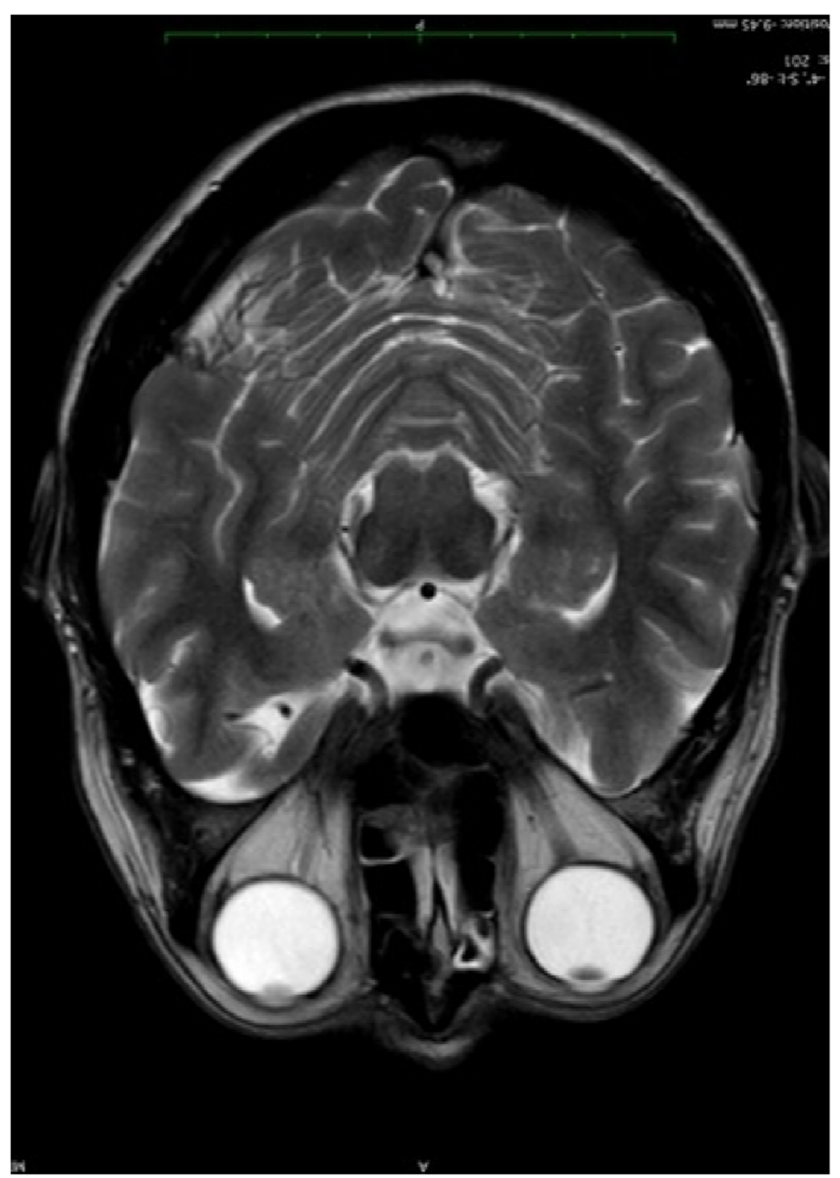

Image 1 MRI axial T2

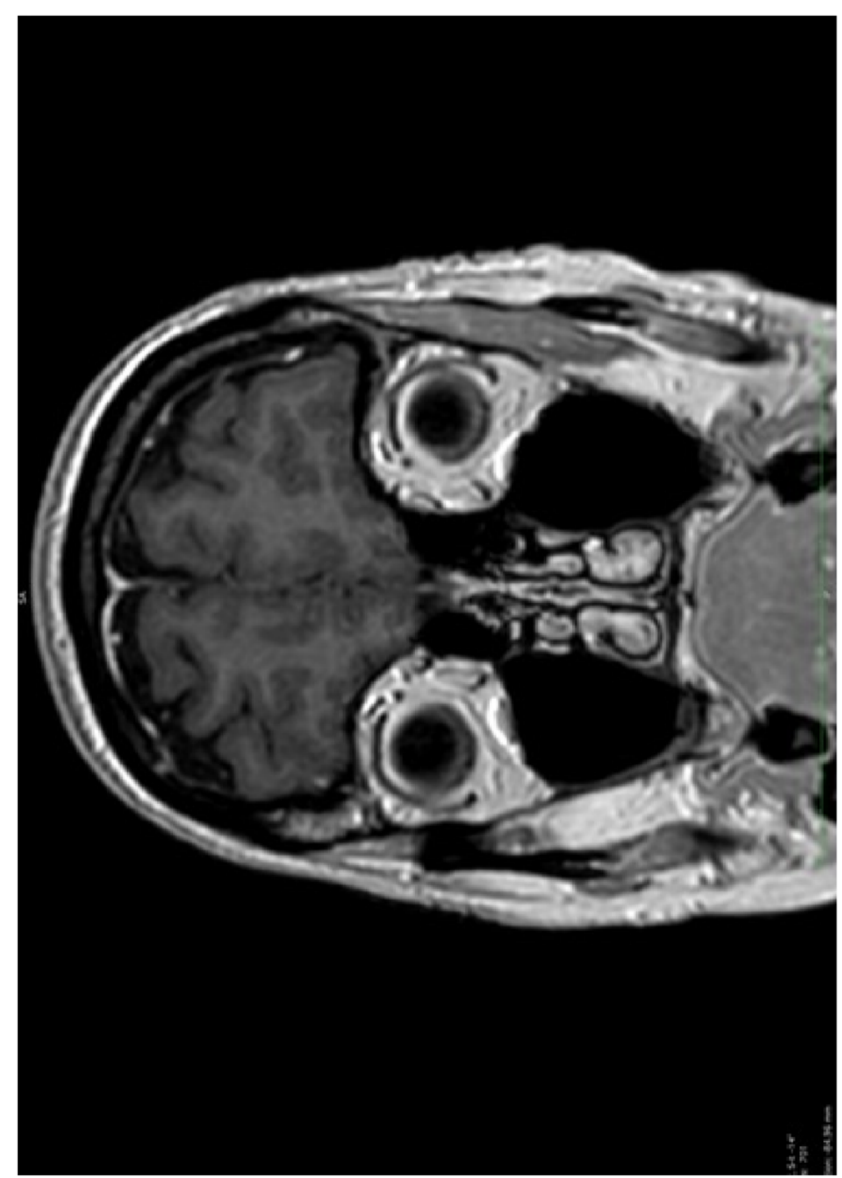

Image 2 MRI Cor T1

\section{Discussion}

In contrast to Galougahi et al., we evaluated symptom status in the late stage of COVID-19 [11].

The smell and taste disorders could mostly be objectified, although not in regard to all patients, which underlines the necessity of objective testing. Compared to Dell'Era et al., who reported a median recovery time of 10 days, our findings show that olfactory impairment is a long-lasting sequela of COVID-19 [16]. Even after 30 to 50 days, patients still suffered from objectifiable smell and taste disorders. In order to rule out permanent damage, further surveillance is necessary.

However, it should be notified that a loss of smell and taste is not pathognomonic for COVID-19, as it appears as well in the course of other respiratory infections. Nevertheless, controlled studies indicate that anosmia is more common in COVID-19 patients than in patients suffering from other viral infections or controls [17]. In contrast to other infectious smell impairments, a loss of smell and taste in COVID-19 seems to be rarely accompanied by a severely blocked nose [18].

The first MRI images showed slight alterations in one patient, which could be associated with a SARS-CoV-2 infection. However, the follow-up examination contradicted this assumption. 


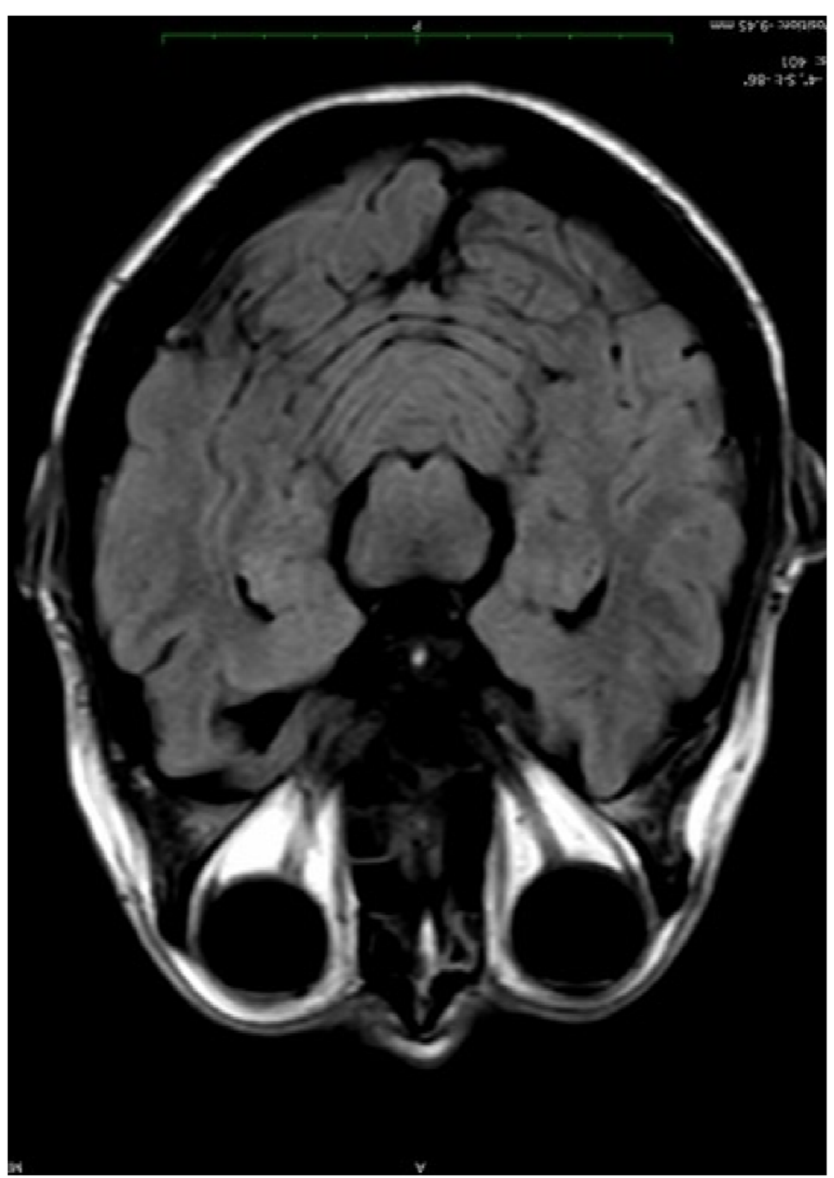

Image 3 MRI axial FLAIR

The MRI investigation in our patients taught us two things: First, at least in chronic stages of the illness, we did not gain any evidence of prolonged neuroinvasive association. Up to now, MRI investigations have focused on the acute stage of illness and are very rare. In our case, the organization was hindered by the concern that such investigations might be an incalculable risk for the staff involved. According to Politi et al., conducting an MRI examination in a patient suffering from persistent severe anosmia and dysgeusia, cortical hyperintensity in the right gyrus rectus could be detected 4 days after symptom onset. Consistent with our results, a complete resolution of the previously seen signal alterations could be observed in a follow-up imaging 28 days after symptom onset [19]. Contrary to assumptions of other researchers, no evidence of a general neuroinvasiveness could be given, at least in the chronic stage of disease [20]. More extensive studies in the acute stage of illness followed by long-term follow-ups are desirable.

Contrary to other evaluations, our study population is rather small which is a limiting factor to the informative value.

As hygiene measures proved to be sufficient avoiding contagion, we encourage medical professionals to conduct specialized examinations and MRIs in the acute stage of disease, which guarantees an optimum patient care.

Authorship Contribution SG and CMS wrote the manuscript and were responsible for the organization. $\mathrm{BH}$ was responsible for Magnetic Resonance Imaging, KF for the objective olfactory and gustatory testing. GW supervised and reviewed the manuscript.

\section{Compliance with Ethical Standards}

Conflict of Interest The authors declare that they have no conflict of interest.

Consent for Publication Written informed consent was obtained from all participants.

Ethics Approval Ethical approval was not necessary as the evaluation was performed in the course of patient care and clinical monitoring.

\section{Appendix}

\begin{tabular}{|c|c|c|c|c|c|c|}
\hline Sequence & TR/TE (msec) & Flip angle degree & FOV (mm) & Matrix & Slice thickness (mm) & No of slices \\
\hline T2W_TSE ax & $6608 / 107$ & 90 & $230 \times 185 \times 170$ & $384 \times 240$ & 5 & 27 \\
\hline sT1W_3D_IR_TFE_tra & $9.4 / 4.6$ & 8 & $250 \times 198 \times 145$ & $252 \times 199$ & 1 & 145 \\
\hline MPR COR & & & & & & 70 \\
\hline MPR SAG & & & & & & 55 \\
\hline FLAIR ax & $8000 / 130$ & 90 & $230 \times 186 \times 153$ & $272 \times 144$ & 4.5 & 28 \\
\hline T2W_FFE ax & $877 / 23$ & 20 & $230 \times 180 \times 148$ & $260 \times 145$ & 4.5 & 25 \\
\hline DWI & $3668 / 85$ & 90 & $230 \times 230 \times 148$ & $128 \times 126$ & 4.5 & 25 \\
\hline KMT1W_3D_MPRAGE & $25 / 4.6$ & 30 & $260 \times 201 \times 175$ & $260 \times 201$ & 1 & 160 \\
\hline KM MPR SAG & & & & & & 50 \\
\hline KM MPR ax & & & & & & 50 \\
\hline $\begin{array}{l}\text { 3D_Brain_VIEW_FLAIR_ } \\
\text { COR }\end{array}$ & $4800 / 332$ & 40 & $250 \times 250 \times 149$ & $208 \times 208$ & 1.2 & 248 \\
\hline 3D_Brain_VIEW_T2 & $2800 / 273$ & 90 & $250 \times 250 \times 138$ & $228 \times 228$ & 1.1 & 250 \\
\hline
\end{tabular}




\section{References}

1. Desforges M, Coupanec AL, Dubeau P, et al. Human coronaviruses and other respiratory viruses: underestimated opportunistic pathogens of the central nervous system? Viruses. 2020;12(1):14.

2. Carod-Artal FJ. Neurological complications of coronavirus and COVID-19. Rev Neurol. 2020;70(9):311-22.

3. Moriguchia T, Hariib N, Goto J, et al. A first case of meningitis/ encephalitis associated with SARS-Coronavirus-2. Int J Infect Dis. 2020;94:55-8.

4. Poyiadji N, Shahin G, Noujaim D, et al. COVID-19-associated acute hemorrhagic necrotizing encephalopathy: CT and MRI features. Radiology. 2020;31:5-30.

5. Baig AM, Khaleeq A, Ali U, Syeda H. Evidence of the COVID-19 virus targeting the CNS: tissue distribution, host-virus interaction, and proposed neurotropic mechanisms. ACS Chem Neurosci. 2020;11:995-8.

6. American Academy of Otolaryngology-Head and Neck Surgery. COVID-19 Anosmia Reporting Tool, American Academy of Otolaryngology- Head and Neck Surgery. May 11, 2020. (https:// www.entnet.org/content/coronavirus-disease-2019-resources).

7. Tudrej B, Sebo P, Lourdaux J, Cuzin C, Floquet M, Haller DM, et al. Self-reported loss of smell and taste in SARS-CoV-2 patients: primary care data to guide future early detection strategies. J Gen Intern Med. 2020;35(8):2502-4.

8. Gane SB, Kelly C, Hopkins C. Isolated sudden onset anosmia in COVID-19 infection. A novel syndrome? Rhinology 2020;58(3): 299-301.

9. Lechien JR, Chiesa-Estomba CM, De Siati DR, et al. Olfactory and gustatory dysfunctions as a clinical presentation of mild-tomoderate forms of the coronavirus disease (COVID-19): a multicenter European study. Eur Arch Otorhinolaryngol. 2020;6:1-11.

10. Vaira LA, Hopkins C, Salzano G, Petrocelli M, Melis A, Cucurullo $\mathrm{M}$, et al. Olfactory and gustatory function impairment in COVID19 patients: Italian objective multicenter-study. Head Neck. 2020;42(7):1560-9.
11. Vaira LA, Deiana G, Fois AG et al. Objective evaluation of anosmia and ageusia in COVID-19 patients: single-center experience on 72 cases. Head \& Neck 2020;42(6):1252-1258.

12. Galougahi MK, Ghorbani J, Bakhshayeshkaram M et al. Olfactory bulb magnetic resonance imaging in SARSCoV-2-induced anosmia: the first report. Acad Radiol 2020;27(6):892-893.

13. Hummel T, Kobal G, Gudziol H, Mackay-Sim A. Normative data for the "Sniffin' sticks" including tests of odor identification, odor discrimination, and olfactory thresholds: an upgrade based on a group of more than 3,000 subjects. Eur Arch Otorhinolaryngol. 2007;264(3):237-43.

14. Landis BN, Welge-Luessen A, Brämerson A, Bende M, Mueller CA, Nordin S, et al. "Taste Strips" - a rapid, lateralized, gustatory bedside identification test based on impregnated filter papers. J Neurol. 2009;256(2):242-8.

15. Helms J, Kremer S, Merdji $\mathrm{H}$ et al. Neurologic features in severe SARS-CoV-2 infection. N Engl J Med 2020;382(23):2268-2270.

16. Dell'Era V, Farri F, Garzaro G, et al. Smell and taste disorders during COVID-19 outbreak: cross-sectional study on 355 patients. Head Neck. 2020;42(7):1591-6.

17. Printza A, Constantinidis J. The role of self-reported smell and taste disorders in suspected COVID-19. Eur Arch Otorhinolaryngol. 2020;277(9):2625-30.

18. Haehner A, Draf J, Dräger S, et al. Predictive value of sudden olfactory loss in the diagnosis of COVID-19. J Oto-RhinoLaryngol. 2020;82(4):175-80.

19. Politi LS, Salsano E, Grimaldi M, et al. Magnetic resonance imaging alteration of the brain in a patient with coronavirus disease 2019 (COVID-19) and anosmia. JAMA Neurol. 2020;77(8):1028-9.

20. Butowt R, Bilinska K. SARS-CoV-2: olfaction, brain infection, and the urgent need for clinical samples allowing earlier virus detection. ACS Chem Neurosci. 2020;11:1200-3.

Publisher's Note Springer Nature remains neutral with regard to jurisdictional claims in published maps and institutional affiliations. 